\title{
Efficacy of two soft-bristle toothbrushes in plaque removal: a randomized controlled trial
}

\author{
Cassiano Kuchenbecker ROSING(a) \\ Juliano CAVAGNI(a) \\ Eduardo José GAIO(a) \\ Francisco Wilker Mustafa Gomes \\ MUNIZ(a) \\ Harry Juan Rivera OBALLE(a) \\ Nicolle RANZAN(a) \\ Stephanie Anagnostopoulos \\ FRIEDRICH(a) \\ Raísa Maldonado SEVERO(a) \\ Elizabeth GITTINS(b) \\ Bernal STEWART(b) \\ Yun Po ZHANG(b) \\ (a) Universidade Federal do Rio Grande \\ do Sul - UFRGS, School of Dentistry, \\ Department of Periodontology, Porto Alegre, \\ RS, Brazil. \\ (b) Colgate-Palmolive Technology Center, \\ Piscataway, NJ, USA.
}

Declaration of Interests: This study was sponsored by Colgate Palmolive Company. Elisabeth Gittins, Bernal Stewart and Yun Po Zhang are currently employed by Colgate Palmolive Company. The other authors are independent researchers.

\section{Corresponding Author:}

Cassiano Kuchenbecker Rösing

E-mail: ckrosing@hotmail.com

DOI: 10.1590/1807-3107BOR-2016.vol30.0134

Submitted: Jul 6, 2016

Accepted for publication: Oct 10, 2016

Last revision: Oct 25,2016
Abstract: The aim of this study was to compare the efficacy in supragingival plaque removal of two soft-bristle toothbrushes. Seventy volunteers were allocated randomly to the Colgate SlimSoft or Curaprox CS5460 toothbrush grourps. At baseline appointment, volunteers underwent plaque examination using the Rustogi Modification of the Navy Plaque Index. Under supervision, they then brushed their teeth for 1minute with their assigned toothbrushes and the plaque examination was repeated. Volunteers performed daily oral hygiene with their assigned toothbrush and a regular dentifrice provided by the researchers for 7 days. The baseline experimental procedures were then repeated. Separate analyses of variance were performed for the whole-mouth, interproximal, and gumline plaque scores $(p<0.05)$. No difference in baseline pre-brushing scores was found between groups. After a single toothbrushing, the mean plaque score was significantly reduced in both groups $(p<0.05)$, with greater reduction of whole-mouth and interproximal plaque scores observed in the SlimSoft group compared with the Curaprox group $(p<0.05)$. After 7 days, the SlimSoft group showed greater reduction of the whole-mouth and interproximal plaque scores compared with the Curaprox group $(\mathrm{p}<0.05)$. In conclusion, the SlimSoft toothbrush presented greater efficacy in supragingival plaque removal than did the Curaprox CS5460 toothbrush, as reflected by whole-mouth and interproximal plaque scores.

Keywords: Toothbrushing; Dental devices, Home care; Dental plaque; Oral hygiene.

\section{Introduction}

Supragingival plaque removal is considered to be one of the most important health promotion strategies in dentistry. It has been considered to be of utmost importance in the decline of the prevalence of caries (especially with the concomitant use of fluoride dentifrices), as well as gingivitis and periodontitis. ${ }^{1}$

Longitudinal studies have consistently demonstrated that good standards of oral hygiene and regular maintenance reduce the incidence of caries, periodontal disease, and tooth loss, and, more recently, that they improve the outcomes of treatment with dental implants..$^{2,3,4,5} \mathrm{~A}$ classical study related to this topic was performed in Sweden; it demonstrated that a strict oral hygiene regimen reduced tooth loss, the number of new decayed surfaces, and periodontal attachment loss. ${ }^{3}$ Other studies have 
also demonstrated the effects of supragingival plaque control on different oral health parameters, reinforcing its importance as the core of preventive dentistry. ${ }^{6}$

Although the importance of supragingival plaque control is generally recognized, oral hygiene practices demand time, dexterity, and motivation; these factors limit the clinical effectiveness of self-performed oral hygiene. Clinical studies have documented the presence of remaining biofilm despite good levels of plaque control. ${ }^{78}$

The consumption of oral hygiene products has increased worldwide. For example, less than one toothbrush per capita was consumed in the 1990s in Brazil. ${ }^{9}$ In 2010, consumption had practically doubled..$^{10}$ Importantly, however, increased consumption does not necessarily translate to better clinical results in terms of effective plaque control.

Toothbrushing is the most widely used method for plaque control, and a wide variety of toothbrushes is available in the market. Studies comparing the efficacy of available toothbrushes are scarce. The use of soft bristles has been recommended to improve plaque reduction while minimizing harm to the gingival tissues. Therefore, comparisons of available instruments are necessary to provide better support for the indication of any given toothbrush. To our knowledge, no study has compared the efficacy of two available soft-bristle toothbrushes - SlimSoft (Colgate-Palmolive Co., New York, USA) and Curaprox CS5460 (Curaden AG, Kriens, Switzerland). The aim of this study was to compare the efficacy of these toothbrushes in plaque removal. The pre-established hypothesis was that no difference in efficacy would be found between the two brushes.

\section{Methodology}

\section{Study design}

This study was designed as a phase III single-center, examiner-blind, two-cell, parallel-group randomized clinical trial.

\section{Ethical aspects}

The Institutional Review Board of the Federal University Rio Grande do Sul approved this study (protocol 1.213.343), and all volunteers signed informed consent forms. The study was conducted according to good clinical practice (GCP).

\section{Sample size estimation}

The sample size calculation utilized historical data from previous studies. Sample size was determined based on a standard deviation for the response measure of 0.12 , an alpha level of 0.05 , and $80 \%$ power. Thirty-five individuals per group were considered to be necessary to detect a minimal statistically significant difference between study groups of $15 \%$.

\section{Subjects}

Seventy healthy adult males and females aged 21-70 years were enrolled in this study. The convenience sample was recruited in October 2015 at the Federal University of Rio Grande do Sul, Brazil. Inclusion criteria comprised good general health; initial mean plaque index $\geq 0.6$, as determined by the Rustogi Modification of the Navy Plaque Index ${ }_{1}^{.11}$ and $\geq 20$ natural uncrowned teeth, excluding third molars. Subjects meeting any of the following criteria were excluded from the study: orthodontic bands, removable partial denture, tumor or significant pathology in the soft or hard tissues of the oral cavity, moderate or advanced periodontal disease (purulent exudate, tooth mobility, and/or extensive loss of periodontal attachment or alveolar bone), five or more carious lesions requiring immediate care, antibiotic use in the month prior to study entry, participation in any other clinical study or test panel within 30 days prior to the start of the study, pregnant or breast-feeding status, dental prophylaxis in the 2 weeks prior to baseline examination, history of allergy to oral/personal care consumer products or their ingredients, use of any prescription medicine that might interfere with the study outcome, medical condition prohibiting abstinence from eating/drinking/chewing gum for 4hours prior to the scheduled visit, and history of alcohol or drug abuse.

\section{Experimental procedures}

Qualifying subjects reported to the clinical study site after refraining from the performance of any oral hygiene procedure for 12 hours and from eating, drinking, or smoking for 4 hours. The baseline 
examination began with evaluation of the soft tissues of the oral cavity and perioral region, followed by plaque disclosure with $6 \mathrm{~mL} 2 \%$ basic fuchsine solution (Eviplac; Biodinâmica, São Paulo, Brazil). A baseline pre-brushing plaque examination was then performed using the Rustogi Modification of the Navy Plaque Index. Supragingival plaque was assessed on the facial and lingual surfaces of each tooth and recorded as present or absent on nine discrete areas of the tooth. From these site-wise scores, a whole-mouth plaque score was determined for each subject by calculating the proportion of sites in the mouth at which plaque was present. The Rustogi Modification of the Navy Plaque Index includes nine areas of each tooth, analyzed from the facial and lingual/palatal aspects. Three areas are near the gingival margin, two are interproximal, and four are on the body of the tooth. This index allows for stratification of area(s) are of concern during the analysis. ${ }^{11}$

Participants were then randomized into two groups of 35 individuals each. The randomization list was computer generated. An external researcher was responsible for allocation concealment. The toothbrushes were kept inside numbered opaque plastic bags. The experimental groups were given:

1. Colgate SlimSoft (Colgate-Palmolive Co.,New York, USA) group;

2. Curaprox CS5460 (Curaden AG, Kriens, Switzerland) group;

Under supervision, the subjects were instructed to brush their teeth for 1 minute with their assigned toothbrush and a commercially available basic fluoride toothpaste (Colgate Cavity Protection; Colgate, São Paulo, Brazil). Baseline post-brushing plaque evaluation was then performed. Subjects were dismissed from the study site with their assigned toothbrush and toothpaste. They were instructed to use the products at home twice daily (morning and evening) for the next 7 days and to refrain from any interproximal cleaning. Subjects returned to the clinical facility for 7-day pre- and post-brushing plaque examinations using the same index and brushing procedure. The same calibrated examiner (CKR), who was unaware of group allocation, performed all plaque examinations.

\section{Statistical analysis}

The main study outcomes were plaque scores, determined using the Rustogi Modification of the Navy Plaque Index. Separate statistical analyses were performed for whole-mouth, interproximal, and gumline plaque scores. A per-protocol analytical method was used. Baseline and 7-day whole-mouth, interproximal, and gumline plaque scores were compared between groups using analysis of variance. Responses were assessed within and between products using mean pre- to post-brushing and pre- to 7-day differences. Within-treatment comparison of baseline and follow-up whole-mouth, interproximal, and gumline plaque scores was performed using paired $t$ tests. All statistical tests were two sided, with a significance level of $a=0.05$. The statistician was blinded to product allocation.

\section{Results}

Three of the 73 subjects screened for study participation were excluded (Figure 1). All 70 randomized subjects completed the 7-day clinical study, and their data were included in the analysis. The two groups did not differ in terms of gender, mean age, or mean baseline plaque score (Table).

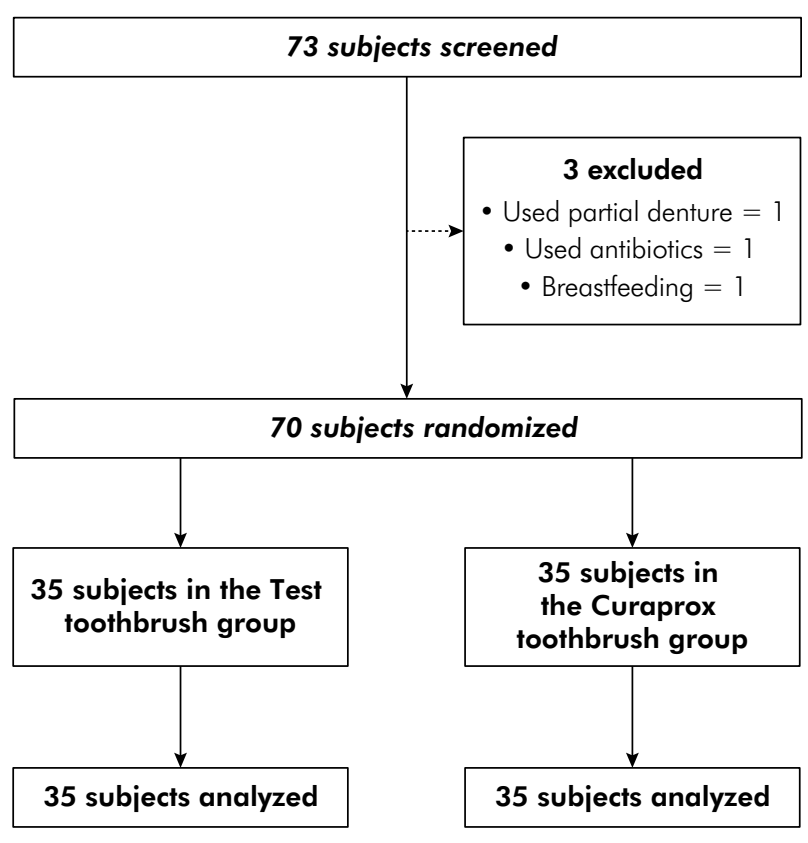

Figure 1. Flow chart of the study. 
Baseline pre-brushing whole-mouth, interproximal, and gumline plaque scores did not differ between groups (Figure 2). A single toothbrushing reduced all of these scores significantly in both groups $(\mathrm{p}<0.05)$. The SlimSoft group showed significantly greater reduction in the mean whole-mouth and interproximal plaque scores in comparison with the Curaprox group ( $p<0.001$; Figure 2). The reduction in the gumline plaque score did not differ between groups.

At the 7-day assessment, toothbrushing significantly reduced the whole-mouth, interproximal, and gumline plaque scores in both groups $(\mathrm{p}<.05$; Figure 3). Subjects in the SlimSoft group exhibited significantly greater reduction in the whole-mouth and interproximal plaque scores compared with

Table. Demographical and baseline characteristics of the study subjects.

\begin{tabular}{lccc}
\hline Variable & SlimSoft group & Curaprox group & $p$-value \\
\hline Male/Female & $13 / 22$ & $19 / 16$ & 0.149 \\
$\begin{array}{l}\text { Mean age } \pm \text { SD } \\
\text { (range) }\end{array}$ & $\begin{array}{c}31.89 \pm 10.21 \\
(21-62)\end{array}$ & $\begin{array}{c}33.26 \pm 12.47 \\
(21-63)\end{array}$ & 0.616 \\
$\begin{array}{l}\text { Mean plaque } \pm \text { SD } \\
\text { (whole-mouth) }\end{array}$ & $0.76 \pm 0.07$ & $0.74 \pm 0.06$ & 0.248 \\
\hline
\end{tabular}

SD: Standard deviation. subjects in the Curaprox group ( $\mathrm{p}<0.001)$, with no significant difference in the gumline plaque score.

During the entire study, five adverse events (one in the SlimSoft group and four in the Curaprox group) were recorded. All five subjects completed the 7-day study. The adverse event reported in the SlimSoft group was tooth sensitivity; the events reported in the Curaprox group were a burning sensation in the mucosa, gingival sensitivity and redness in the upper arch, gingival bleeding for 2 hours after brushing, and development of aphtha in the upper arch.

\section{Discussion}

The present randomized controlled clinical trial compared the efficacy in plaque removal of two commercially available toothbrushes. The use of the SlimSoft toothbrush resulted in greater plaque reduction than did the use of the Curaprox toothbrush, as demonstrated by whole-mouth and interproximal plaque scores. The trial was designed according to contemporary clinical epidemiological paradigms and following GCP standards. The report is based on the CONSORT statement.

The results of this study should be considered within the perspective of its design, which aimed to verify efficacy. They should be understood as reflecting the capacity of the toothbrushes to remove dental

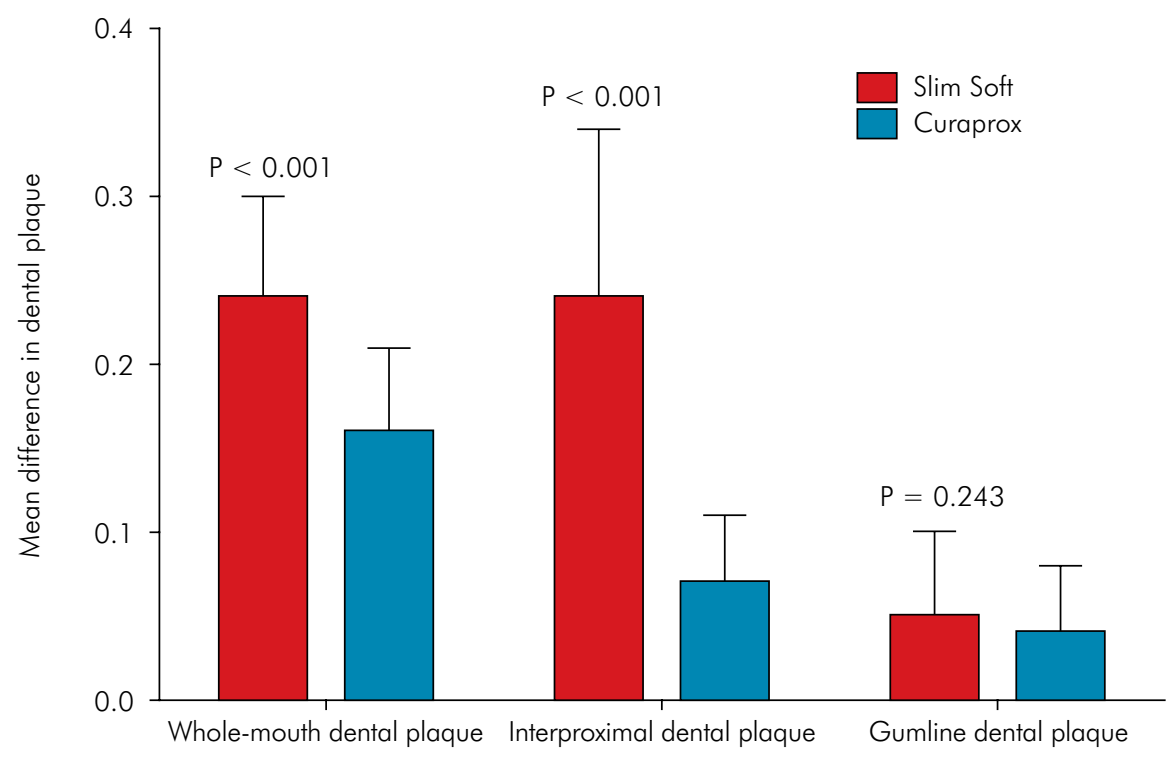

Figure 2. Mean differences in plaque scores from baseline pre-brushing to baseline post-brushing. p-values refer to inter-group comparisons (analysis of variance). 


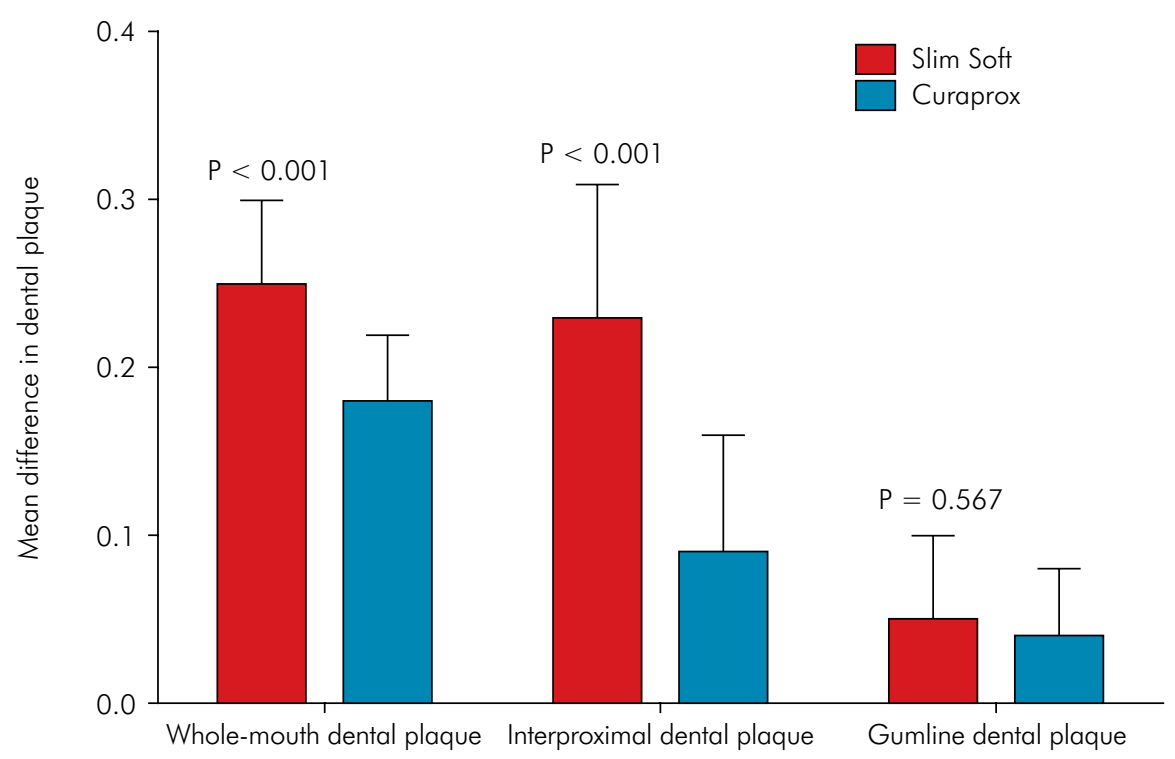

Figure 3. Mean differences in plaque scores from 7-day pre-brushing to 7-day post-brushing. $P$ values refer to inter-group comparisons (analysis of variance).

plaque. The study outcomes were measured using the Rustogi Modification of the Navy Plaque Index, ${ }^{11}$ which enables the analysis of plaque topography in accordance with the American Dental Association's guidelines for toothbrush studies. ${ }^{12}$ We calculated whole-mouth, gumline, and interproximal plaque scores. In addition, plaque examinations were conducted at two experimental timepoints (baseline and 7 days) to assess whether a learning curve affected the results. The same pattern of plaque removal was observed in both groups, independent of the timepoint. Comparison between baseline pre-brushing and post-brushing scores, and 7-day pre-brushing and post-brushing scores revealed the same trend. The lack of a difference in pre-brushing plaque scores between groups indicates that randomization was effective. In addition, the amount of plaque accumulation resulting from the 12-hour periods of refraining from oral hygiene before both evaluations was sufficient to test the efficacy of the toothbrushes.

The null hypothesis that no difference in the efficacy of plaque removal would be observed between toothbrushes was rejected in two analyses (whole-mouth and interproximal plaque scores), the results of which favored the SlimSoft toothbrush. The reduction in the whole-mouth plaque score was $35-50 \%$ greater in the SlimSoft group than in the
Curaprox group. The whole-mouth plaque score is a useful measure of the potential of a toothbrush to remove plaque as a whole, which is of great value for the assessment of clinical efficacy. It has been used in previous studies to infer the cleaning potential of toothbrushes. ${ }^{13}$ The most interesting result of this study concerns the interproximal plaque score. Studies have clearly demonstrated that the interproximal areas are critical, as the occurrence of gingival inflammation is greater in these areas. ${ }^{14}$ Although toothbrushing focuses on the buccal and lingual tooth surfaces, this study showed that it achieved a substantial reduction in interproximal plaque, even without the use of an interdental cleaning device. This finding is of clinical importance, as flossing is not widespread. Therefore, it is interesting that a toothbrush can reach interproximal areas. The reduction of interproximal plaque achieved with the SlimSoft toothbrush was 50-242\% greater than that achieved with the Curaprox toothbrush. This result could be attributable to the design of the toothbrush. Participants refrained from interproximal cleaning during the study period, and compliance with this instruction was checked during follow-up visits.

None of the five adverse events reported in the present study was related to the protocol. The burning sensation, eventual bleeding, and hypersensitivity 
might be related to a study participation effect, in which individuals may have brushed more vigorously. The use of only 1 minute brushing, as in a previous study, also aimed to avoid excessive brushing.

This study has strengths and limitations that should be noted. Among the strengths is the study design, with randomization of the participants, use of a standardized brushing time, examiner reproducibility, blinding of the examiner and statistician, and $100 \%$ compliance with no dropout. The limitation is that effectiveness was not assessed due to the short duration of the study. In addition, the comparative

\section{References}

1. Chapple IL, Van der Weijden F, Doerfer C, Herrera D, Shapira L, Polak D et al. Primary prevention of periodontitis: managing gingivitis. J Clin Periodontol. 2015;42(Suppl 16 ):S71-6. doi:10.1111/jcpe.12366

2. Jepsen S, Berglundh T, Genco R, Aass AM, Demirel K, Derks J et al. Primary prevention of peri-implantitis: managing peri-implant mucositis. J Clin Periodontol. 2015;42(Suppl 16):S152-7. doi:10.1111/jcpe.12369

3. Axelsson P, Nyström B, Lindhe J. The long-term effect of a plaque control program on tooth mortality, caries and periodontal disease in adults: results after 30 years of maintenance. J Clin Periodontol. 2004;31(9):749-57. doi:10.1111/j.1600-051X.2004.00563.x

4. Hirschfeld L, Wasserman B. A long-term survey of tooth loss in 600 treated periodontal patients. J Periodontol. 1978;49(5):225-37. doi:10.1902/jop.1978.49.5.225

5. Lindhe J, Nyman S. Long-term maintenance of patients treated for advanced periodontal disease. J Clin Periodontol. 1984;11(8):504-14. doi:10.1111/j.1600-051X.1984.tb00902.x

6. Rosling B, Nyman S, Lindhe J, Jern B. The healing potential of the periodontal tissues following different techniques of periodontal surgery in plaque-free dentitions: a 2-year clinical study. J Clin Periodontol. 1976;3(4):233-50. doi:10.1111/j.1600-051X.1976.tb00042.x

7. Haas AN, Castro GD, Moreno T, Susin C, Albandar JM, Oppermann RV et al. Azithromycin as an adjunctive treatment of aggressive periodontitis: 12-months randomized clinical trial. J Clin Periodontol. 2008;35(8):696-704. doi:10.1111/j.1600-051X.2008.01254.x effect of such brushes on gingival inflammation remains to be studied.

\section{Conclusion}

In conclusion, the SlimSoft toothbrush showed greater efficacy in plaque removal than did the Curaprox toothbrush, as demonstrated by whole-mouth and interproximal plaque scores.

\section{Acknowledgments}

The present study was supported by Colgate Palmolive Inc.
8. Moreira CH, Weidlich P, Fiorini T, Rocha JM, Musskopf ML, Susin C et al. Periodontal treatment outcomes during pregnancy and postpartum. Clin Oral Investig. 2015;19(7):1635-41. doi:10.1007/s00784-014-1386-Z

9. Gjermo P, Rösing CK, Susin C, Oppermann R. Periodontal diseases in Central and South America. Periodontol 2000. 2002;29(1):70-8. doi:10.1034/j.1600-0757.2001.290104.x

10. Oppermann RV, Haas AN, Rösing CK, Susin C. Epidemiology of periodontal diseases in adults from Latin America. Periodontol 2000. 2015;67(1):13-33. doi:10.1111/prd.12061

11. Rustogi KN, Curtis JP, Volpe AR, Kemp JH, McCool JJ, Korn LR. Refinement of the Modified Navy Plaque Index to increase plaque scoring efficiency in gumline and interproximal tooth areas. J Clin Dent. 1992;3(C Suppl):C9-12.

12. American Dental Association - ADA. Acceptance program guidelines: toothbrushes. Chicago: American Dental Association; 2009.

13. Ghassemi A, Vorwerk L, Hooper W, Patel V, Milleman JL, Milleman KR. Comparative plaque removal efficacy of two new powered toothbrushes and a manual toothbrush. J Clin Dent. 2014;25(2):1-5.

14. Hugoson A, Koch G. Oral health in 1000 individuals aged 3--70 years in the community of Jönköping, Sweden: a review. Swed Dent J. 1979;3(3):69-87.

15. Sharma NC, Qaqish J, Walters PA, Grender J, Biesbrock AR. A clinical evaluation of the plaque removal efficacy of five manual toothbrushes. J Clin Dent. 2010;21(1):8-12. 


\section{Efficacy of two soft-bristle toothbrushes in plaque removal: a randomized controlled trial. Braz. Oral Res. 2016, vol.30, n.1, e134.}

Declaration of interest:

Where is read: Declaration of Interest: The authors certify that they have no commercial or associative interest that represents a conflict of interest in connection with the manuscript.

\section{It should read:}

Declaration of Interest: This study was sponsored by Colgate Palmolive Company. Elisabeth Gittins, Bernal Stewart and Yun Po Zhang are currently employed by Colgate Palmolive Company. The other authors are independent researchers.

DOI: 10.1590/1807-3107BOR-2016.vol30.0134erratum 